\title{
Evaluation of the olfactory bulb volume and morphology in patients with coronavirus disease 2019: can differences create predisposition to anosmia?
}

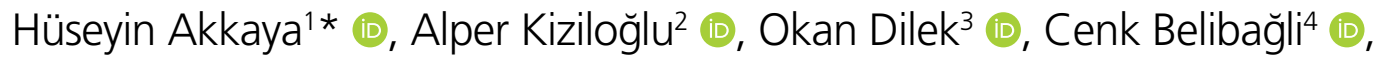 \\ Ömer Kaya ${ }^{5}$ (D), Cengiz Yılmaz³ ${ }^{3}$, Bozkurt Gülek ${ }^{3}$ (D)
}

\section{SUMMARY}

OBJECTIVE: This study aimed to investigate whether the volume and morphology of the olfactory bulb are effective in the occurrence of anosmia in patients after COVID-19 infection.

METHODS: The olfactory bulbus volume was calculated by examining the brain magnetic resonance imaging of cases with positive (+) COVID-19 polymerase chain reaction test with and without anosmia. Evaluated magnetic resonance imaging images were the scans of patients before they were infected with COVID-19. The olfactory bulbus and olfactory nerve morphology of these patients were examined. The brain magnetic resonance imaging of 59 patients with anosmia and 64 controls without anosmia was evaluated. The olfactory bulb volumes of both groups were calculated. The olfactory bulb morphology and olfactory nerve types were examined and compared between the two groups.

RESULTS: The left and right olfactory bulb volumes were calculated for the anosmia group and control group as $47.8 \pm 15 / 49.3 \pm 14.3$ and $50.5 \pm 9.9 / 50.9 \pm 9.6$, respectively. There was no statistically significant difference between the two groups. When the olfactory bulb morphology was compared between the two groups, it was observed that types $D$ and $R$ were dominant in the anosmia group ( $p<0.05$ ). Concerning olfactory nerve morphology, type $\mathrm{N}$ was significantly more common in the control group $(\mathrm{p}<0.05)$.

CONCLUSIONS: According to our results, the olfactory bulb volume does not affect the development of anosmia after COVID-19. However, it is striking that the bulb morphology significantly differs between the patients with and without anosmia. It is clear that the evaluation of COVID-19-associated smell disorders requires studies with a larger number of patients and a clinicoradiological approach. KEYWORDS: COVID-19. Anosmia. Olfactory bulb. Olfactory nerve. Olfactory mucosa. Epithelium.

\section{INTRODUCTION}

Although coronavirus mainly targets the respiratory system, it can also spread from the respiratory tract to the central nervous system due to its neuroinvasive ability ${ }^{1}$. Therefore, patients with coronavirus disease 2019 (COVID-19) may present with a variety of neurological symptoms such as ischemic infarction, meningitis, encephalitis, bleeding, acute hemorrhagic necrotizing encephalopathy, cerebral venous thrombosis, and diffuse leukoencephalopathy with microhemorrhage ${ }^{1-4}$. Olfactory dysfunction starts suddenly in most cases and is usually temporary, with the recovery time ranging from 1-3 weeks 5 .

\footnotetext{
'Siverek State Hospital, Department of Radiology - Sanliurfa, Turkey. 2Develi State Hospital, Department of Radiology - Kayseri, Turkey.

${ }^{3}$ University of Health Sciences, Adana Training and Research Hospital, Department of Radiology - Adana, Turkey.

${ }^{4}$ University of Health Sciences, Adana Training and Research Hospital, Department of Family Medicine - Adana, Turkey.

${ }^{5}$ Cukurova University, Faculty of Medicine, Department of Radiology - Adana, Turkey.

*Corresponding author: dr.hsynakkaya@gmail.com

Conflicts of interest: the authors declare there are no conflicts of interest. Funding: none.

Received on July 16, 2021. Accepted on August 14, 2021.
} 
Chemosensory symptoms may manifest as viral prodromes or codevelop with other disease symptoms. However, a significant relationship between COVID-19 and sinonasal symptoms has not yet been detected, suggesting that the pathogenesis of anosmia may differ from obstructive olfactory dysfunction that is seen in other viral upper respiratory tract infections $s^{6,7}$.

The olfactory bulb (OB) is located above the cribriform plate just below the olfactory sulcus (OS) in the anterior cranial fossa and is easily recognizable on conventional magnetic resonance imaging (MRI). The olfactory neural network is connected to the piriform cortex and amygdala through first-order projections and to the orbitofrontal cortex, thalamus, and insula through secondary projections ${ }^{8,9}$.

There is limited literature on OB imaging in COVID-19 olfactory dysfunction, with the availability of only a few case reports ${ }^{10,11}$. Abnormal findings reported include microhemorrhage in $\mathrm{OB}$, signal abnormality, increased enhancement, and enlarged or reduced atrophied $\mathrm{OB}^{9,12}$.

To the best of our knowledge, there is no study in the English language literature evaluating the effect of $\mathrm{OB}$ and OS morphology on the frequency of anosmia in patients with COVID-19. This study aimed to determine whether the OB volume and $\mathrm{OB}$ and $\mathrm{OS}$ morphologies were associated with the frequency of anosmia in COVID-19-positive patients.

\section{METHODS}

\section{Patient selection}

This study was approved by the Ethical Committee and conducted in full accordance with the guidelines of the Declaration of Helsinki. This study was retrospective, and 7,538 patients who were admitted to our hospital's COVID Outpatient Clinic between April 2020 and December 2020 and who were positive for COVID-19 according to the polymerase chain reaction test (PCR) were screened. A total of 123 patients who had brain MRI before the development of anosmia were included in this study. Patients with brain MRI examination older than one year were excluded from this study, as the reliability of clinical information may be impaired.

Out of these, 59 patients, whose olfactory dysfunction still persisted despite the improvement of other COVID-19-related symptoms, were selected for the anosmia group.

There were no other reasons that could cause smell disorders in the patients in the anosmia group. Patients with a history of neurodegenerative disease were excluded from this study considering that their anosmia might not have been associated with COVID-19 $9^{13,14}$. In addition, patients with a Kennedy staging of other than 0 according to the paranasal sinus MRI examination and those with a history of chronic rhinosinusitis were excluded since these conditions could cause anosmia ${ }^{15,16}$. Finally, pediatric patients, pregnant women, patients with a previous history of loss or changes in smell and taste, and those with allergic rhinitis, a history of head and neck trauma or migraine, and pathological signal changes in the amygdala and orbitofrontal cortex on brain MRI were also excluded from this study. As the control group, 64 people who did not complain of loss of smell during or after COVID-19 and who had brain MRI in the last year due to nonspecific headache and vertigo were selected.

\section{MRI acquisition}

MRI examination was performed on a 1.5 T unit (Philips Ingenia, Best, Eindhoven, Netherlands, 2017). The technical parameters were as follows: axial T1-weighted [repetition time (TR): 550-750 ms; echo time (TE): 20-25 ms; scan thickness: $5 \mathrm{~mm}$; slice gap: $1 \mathrm{~mm}$; and matrix: $256 \times 256$ ], axial T2-weighted (TR: 4,000-5,000 ms; TE: 90-120 ms; scan thickness: $5 \mathrm{~mm}$; slice gap: $1 \mathrm{~mm}$; and matrix: $256 \times 256$ ), sagittal T2-weighted fluid-attenuated inversion recovery (FLAIR) (TR: , 7,200 ms; TE: $120 \mathrm{~ms}$; FA: $90^{\circ}$; TI: 1,333-2,041 ms; and matrix, 256×256), and coronal T2 (TR: 6,550 ms; TE: $99 \mathrm{~ms}$, flip angle: $150^{\circ}$; slice thickness: $5 \mathrm{~mm}$; and matrix: $256 \times 256$ ). All images were evaluated using the Philips IntelliSpace workstation.

\section{MRI evaluation}

The OS depth was measured on coronal T2 images to the deepest point of the OS by drawing a tangent line to the lower boundaries of the gyrus rectus and medial orbital gyrus ${ }^{17}$. The OB volume and morphology were evaluated in coronal T2-weighted sections. Since most pathologies can affect the ipsilateral gyrus rectus and $\mathrm{OB}$ simultaneously, the reference point was taken as the corticomedullary signal intensity of the contralateral gyrus rectus. The oval or inverted J-shaped OBs were considered normal (type N) (Figure 1A). Shrunken or flattened OBs without deformity were accepted as type $\mathrm{R}^{18,19}$ (Figures $1 \mathrm{~B}$ and 1D). The presence of asymmetric contour lobulation or hyperintense focus of $>1$ on T2 images was accepted as type $\mathrm{D}^{17-19}$ (Figure 1C). The olfactory nerve was evaluated using the sagittal FLAIR sequence. Thin and straight stretched fibers that were evenly aligned were considered normal (type N) ${ }^{18-20}$ (Figures 2A and $2 \mathrm{~B}$ ). Non-uniform olfactory nerves with an irregular inferior projection in the lower contour of $\mathrm{OB}$ were considered as type $\mathrm{C}$ while those with markedly thinned calibration were classified as thinning and scarcity (TS) ${ }^{19-21}$ (Figures 2C and 2D). The evaluations were performed based on the consensus of three radiologists, blinded to the clinical information of the patients. In case of disagreement, an experienced radiologist's opinion was sought. 


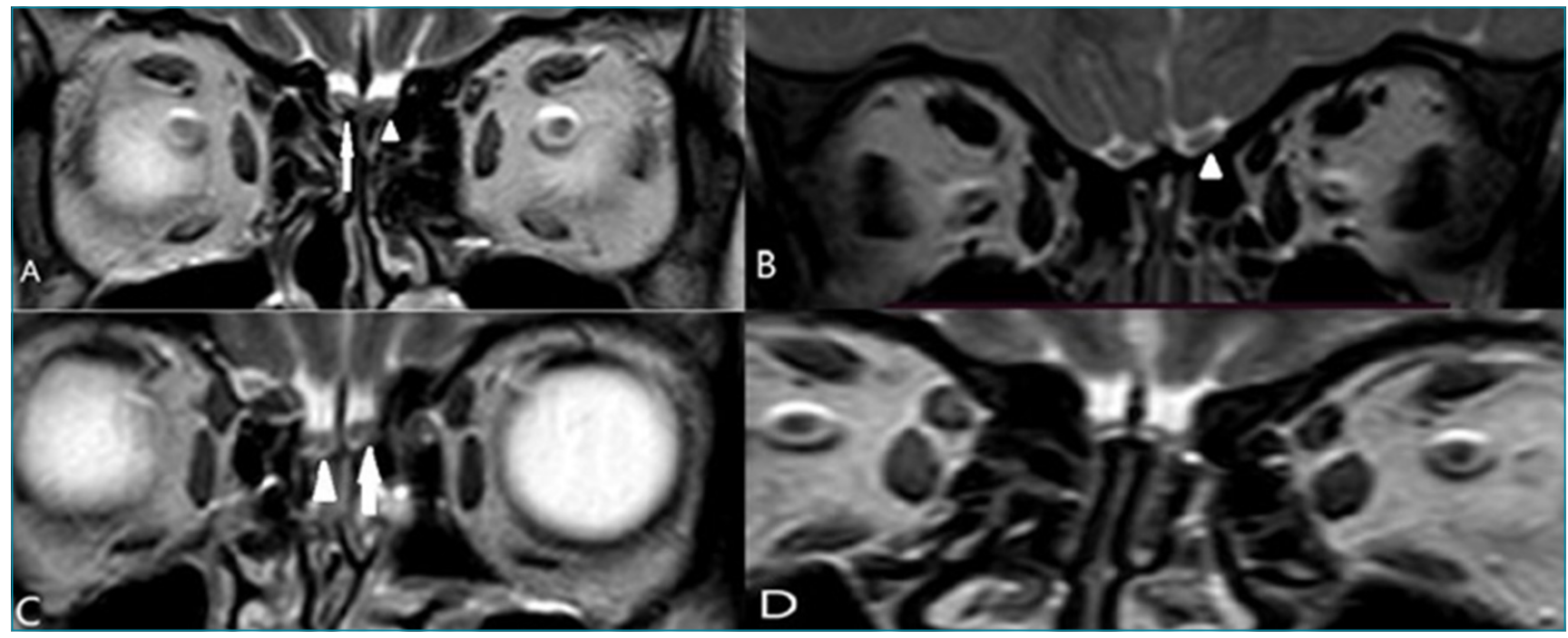

Figure 1. (A and B): Coronal T2-weighted examination shows that the right olfactory bulbus is seen as inverted $\mathrm{j}$ and was considered to be normal type (long arrow), and the left olfactory bulbus appears flattened and shrunken and was considered type R (arrowhead). (C): Coronal T2-weighted examination shows that the right olfactory bulbus shows more than 1 hyperintense focus and asymmetric contour lobulation (arrowhead), and the left olfactory bulbus shows contour lobulation (arrow) both were considered type D. (D): Coronal T2-weighted examination shows that both olfactory bulbus were considered flattened and type R.

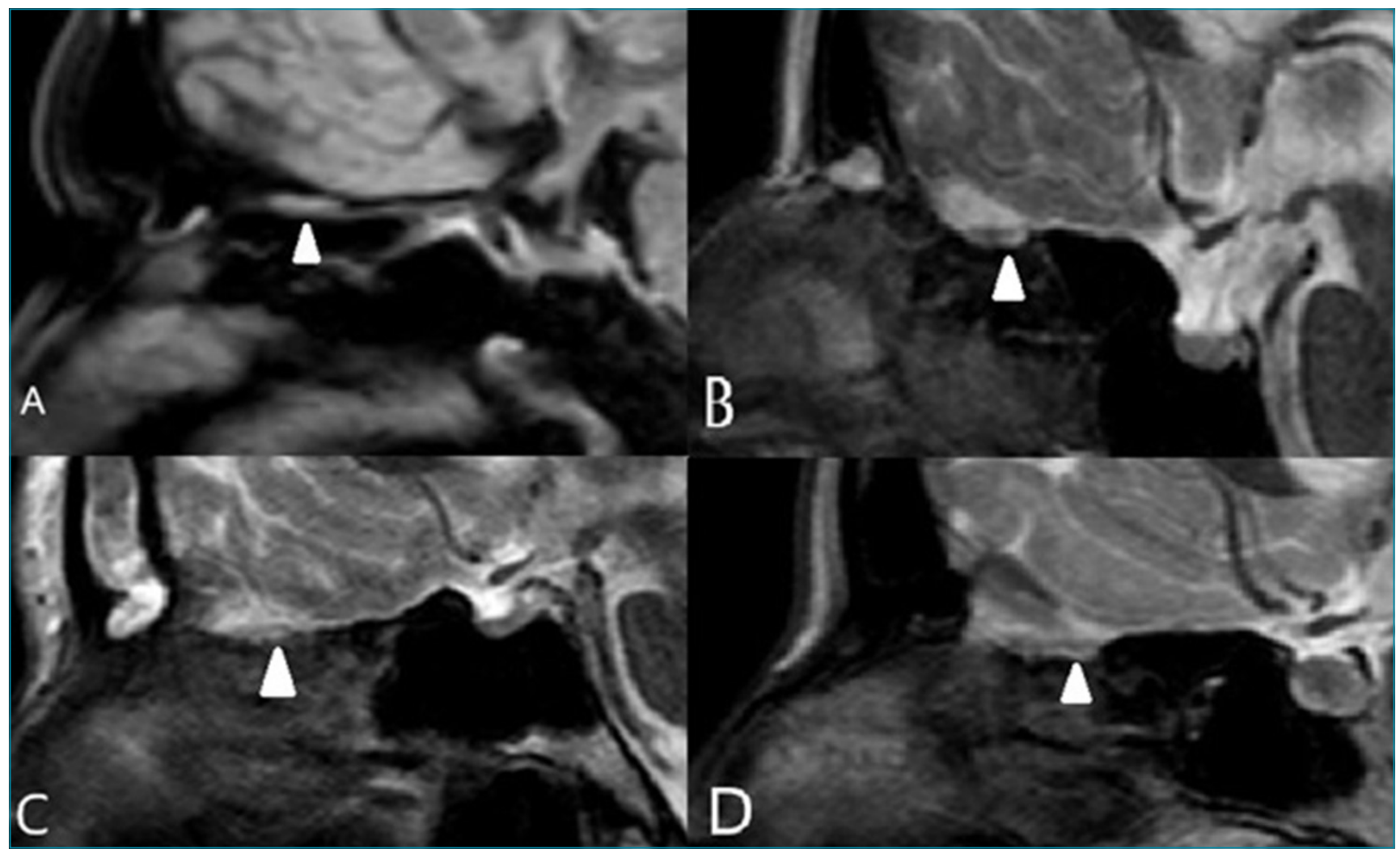

Figure 2. (A): Sagittal fluid-attenuated inversion recovery and (B): saggital T2-weighted examination show that smooth and thick olfactory nerve was accepted as type N. (C): Saggital T2-weighted examination shows that the significantly thinned and deformed olfactory nerve was accepted as type thinning and scarcity (olfactory nerves marked with arrowhead). (D): Sagittal fluid-attenuated inversion recovery weighted examination shows that nonuniform olfactory nerve with thinned and deformed appearance was accepted as type thinning and scarcity (olfactory nerves marked with arrowhead). 


\section{Statistical analysis}

The Statistical Package for the Social Sciences (SPSS) version 23.0 software package was used for the statistical analysis of the data. Categorical measurements were summarized as numbers and percentages and continuous measurements as mean and standard deviation values (median and minimum-maximum where necessary). The Shapiro-Wilk test was used to determine whether the parameters in this study showed a normal distribution. In the comparison of continuous measurements between the groups, the normality of distribution was checked, the Mann-Whitney $U$ test was used in binary variables for parameters that did not show a normal distribution, and the independent Student's $t$-test was used for paired group analyses for normally distributed data. The statistical significance level was 0.05 in all tests.

\section{RESULTS}

In this study, a total of 123 brain MRIs taken before the COVID19 PCR (+) positivity, 59 belonging to the anosmia group and 64 to the control group, were retrospectively analyzed. There were 33 men and 26 women in the anosmia group and 33 men and 31 women in the control group. The mean age was 54.5 (21-71) years for the anosmia group and 55 (19-80) years for the control group. There was no statistically significant difference in age and gender between the two groups ( $p=0.29$ and 0.627 , respectively) (Table 1 ).

No statistically significant difference was found between the anosmia and control groups in relation to the left and right OB volumes ( $\mathrm{p}=0.236$ and 0.467 , respectively). Similarly, there was no statistically significant difference in the left and right OS depths between the two groups ( $\mathrm{p}=0.92$ and 0.374 , respectively) (Table 1; Figure 3).

Table 1. Statistical analysis of the demographic data and olfactory bulb and olfactory bulb parameters of the study groups.

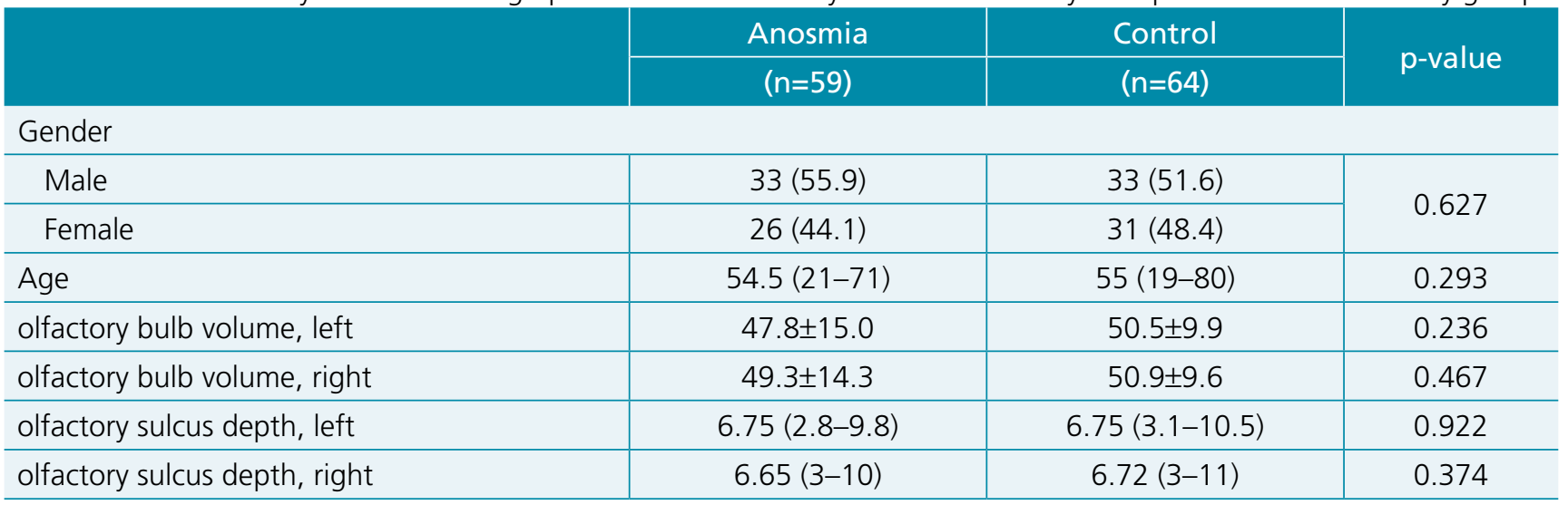

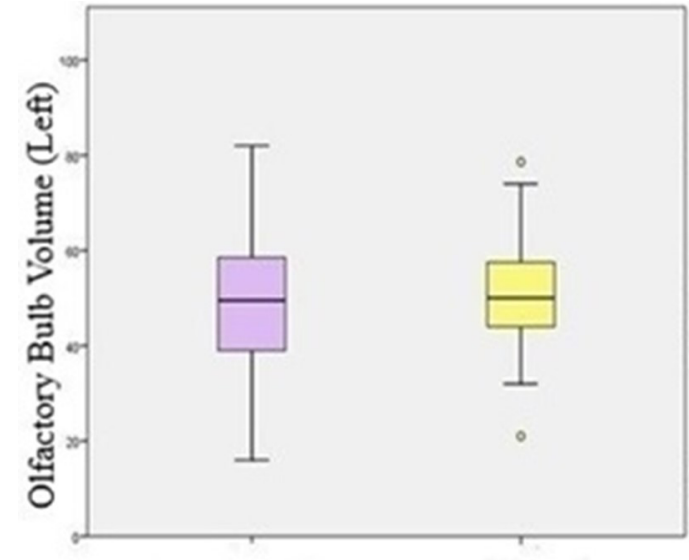

Anosmia
Controls

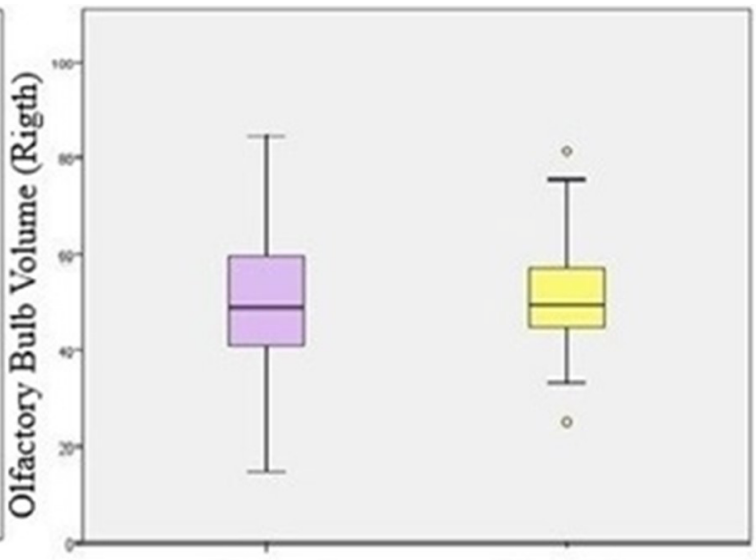

Anosmia

\section{Controls}

Figure 3. Box and whisker plots showing the median and interquartile range values for each group. No statistically significant difference was found between anosmia and control groups in terms of olfactory bulbus volumes. 
When the OB types were compared between the anosmia and control groups, types $\mathrm{D}$ and $\mathrm{R}$ were more common in the anosmia group with a statistically significant difference. Type J was found at a higher rate in the control group compared with the anosmia group, and this was at a statistically significant level (Table 2).

When the distribution of olfactory nerve morphology was examined in both groups, type $\mathrm{N}$ was more common in the control group at a statistically significant rate. In contrast, the rates of patients with types $\mathrm{C}$ and TS did not differ between the anosmia and control groups (Table 2).

\section{DISCUSSION}

The pathogenesis of olfactory dysfunction in COVID-19 disease is not yet fully understood; however, studies have shown no significant association between sinonasal symptoms and COVID-1922. According to a hypothesis, in COVID-19, anosmia is caused by the virus entering the central nervous system through olfactory sensory neurons in the olfactory mucosa ${ }^{23}$. It has also been previously shown that COVID-19 can migrate from the nose to OB in an experimental mouse model ${ }^{24}$. The possible mechanisms that are most frequently considered

Table 2. Statistical distribution and analysis of olfactory bulb and olfactory nerve types in the study groups.

\begin{tabular}{|c|c|c|c|c|}
\hline & \multirow{2}{*}{$\begin{array}{c}\text { Anosmia } \\
(n=59)\end{array}$} & \multirow{2}{*}{$\frac{\text { Control }}{(n=64)}$} & \multirow{2}{*}{ p-value } \\
\hline & & & & \\
\hline \multicolumn{5}{|c|}{ olfactory bulb types, left } \\
\hline & $\mathrm{D}$ & $16(27.1)$ & $8(12.5)$ & 0.041 \\
\hline & J & $22(37.3)$ & $43(67.2)$ & 0.003 \\
\hline & $\mathrm{R}$ & $21(35.6)$ & $13(20.3)$ & 0.045 \\
\hline \multicolumn{5}{|c|}{ olfactory bulb types, right } \\
\hline & D & $15(25.4)$ & $7(10.9)$ & 0.036 \\
\hline & J & $18(30.5)$ & $40(62.5)$ & 0.001 \\
\hline & $\mathrm{R}$ & $26(44.1)$ & $17(26.6)$ & 0.042 \\
\hline \multicolumn{5}{|c|}{ Olfactory nerve types, left } \\
\hline & C & $26(37.3)$ & $19(29.7)$ & 0.098 \\
\hline & TS & $22(18.6)$ & $15(23.4)$ & 0.094 \\
\hline & N & $11(81.4)$ & $30(46.9)$ & 0.001 \\
\hline \multicolumn{5}{|c|}{ Olfactory nerve types, right } \\
\hline & C & $23(35.6)$ & $18(28.1)$ & 0.202 \\
\hline & TS & $21(25.4)$ & $15(23.4)$ & 0.139 \\
\hline & N & $15(74.6)$ & $31(48.4)$ & 0.008 \\
\hline
\end{tabular}

Bold values are statistically relevant. in the pathogenesis of COVID-19 anosmia are olfactory cleft inflammation/occlusion and/or OB damage ${ }^{23,25}$.

Angiotensin-converting enzyme 2 (ACE2) receptors, target molecules for COVID-19, are expressed by non-neuronal support cells of the olfactory epithelium but not directly by olfactory neurons. Anosmia may result from injury to the supporting cells of the epithelium. This is supported by postviral anosmia studies in which prolonged or persistent anosmia reflects the olfactory epithelial regeneration interval ${ }^{24}$.

Evaluating imaging findings in patients with anosmia can be very complex. In such evaluations, $\mathrm{OB}$ volume and $\mathrm{OS}$ depth were considered the most effective and were the most frequently measured parameters ${ }^{21,23}$. Although exact values have not been determined in studies on $\mathrm{OB}$ volume, the common consensus is that the normal $\mathrm{OB}$ volume is $>45 \mathrm{~mL}$, and the normal OS depth is $>7 \mathrm{~mm}^{13,20,22}$.

The literature shows that $\mathrm{OB}$ volume loss is mainly detected in the idiopathic or post-viral anosmia groups ${ }^{11,12,22}$. In this study, no significant difference was found in the pre-disease $\mathrm{OB}$ volumes of the anosmia and control groups. Despite the presence of contradictions in the findings and the lack of a complete consensus in the literature, our results indicate that $\mathrm{OB}$ volume may not really be the main cause of anosmia after COVID-19. Similar studies, prospective studies if possible, with larger series are needed to clarify this issue.

The data in this study showed that the risk of anosmia was increased among the patients with type $\mathrm{D}$ and $\mathrm{R}$ OBs. The $\mathrm{OB}$ volume did not statistically significantly differ between the study groups while the difference in the OB type was statistically significant, suggesting that nerve morphology rather than volume might be effective in anosmia. It is clear that there is still a need to ascertain why the types of OB morphology result in a difference in olfactory function.

In light of these data, our hypothesis is the possibility of differences in primary neurons in the olfactory mucosa accompanying morphological types $\mathrm{D}$ and $\mathrm{R}$ that can be defined by MRI.

When the literature is examined, there is no hypothesis as to why the morphological type is effective in the loss of smell after COVID-19. However, we have a few hypotheses to explain this situation. The strongest of these hypotheses is that nerves may have varied in their morphology as well as the surface areas where they terminate in the cribriform plates. Thus, the relationship between the variability of angiotensin-converting enzyme 2 receptor (ACE2) and transmembrane protease serine two receptor density and morphology can be explained ${ }^{24,25}$. Postmortem autopsy studies are needed to evaluate the validity of this hypothesis, as primary sensory neurons in the olfactory mucosa cannot be visualized by MRI. Another hypothesis is that morphology is important only in the patient group we 
evaluated. Multicenter, multi-participant studies are needed to confirm whether this is coincidental or whether morphology actually has meaning.

When the olfactory nerve types were compared between the anosmia and control groups, types $\mathrm{C}$ and TS, which are less common in the general population, were not statistically associated with olfactory dysfunction. However, type N, which is the most common type and indicates normal morphology, was seen at a significantly higher rate in the control group without anosmia. We consider that similar studies to be conducted with a higher number of patients may present comprehensive data on this subject.

There were some limitations to this study. We consider the major limitation to be the relatively small sample size due to the strict criteria used in patient selection. Other limitations include the retrospective nature of the study and the absence of an interobserver evaluation.

For the diagnosis of anosmia, the complaints of the patients were taken as a basis, and no objective test was used. It would be ideal to use an orbital MRI to evaluate the OB; however, it would be very difficult to achieve this in pandemic conditions. Therefore, brain MRI was used, and this can be considered as another limitation.

\section{CONCLUSIONS}

It is commonly accepted that COVID-19 affects OB, but it has not yet been elucidated how this effect on $\mathrm{OB}$ causes olfactory dysfunction. In this study, we observed that the $\mathrm{OB}$ volume before COVID-19 infection had no significant effect on the etiology of anosmia development during or after the disease. However, it is considered that the morphology of the $\mathrm{OB}$ type and primary neurons in the accompanying olfactory mucosal epithelium may play a key role in olfactory dysfunction, and there is a need for further studies to shed light on this subject.

\section{AUTHORS" CONTRIBUTIONS}

HA: Conceptualization, Data curation, Formal Analysis, Writingoriginal draft, Writing - review \& editing. OD: Conceptualization, Data curation, Formal Analysis, Writing - review \& editing. ÖK: Conceptualization, Data curation, Formal Analysis, Writing review \& editing. CY: Conceptualization, Data curation, Formal Analysis, Writing - review \& editing. CB: Conceptualization, Data curation, Formal Analysis, Writing - review \& editing. AK: Conceptualization, Data curation, Formal Analysis, Writing - review \& editing. BG: Conceptualization, Data curation, Formal Analysis, Writing - review \& editing. CB: Writing - review \& editing.

\section{REFERENCES}

1. Acar T, Acar BA, Aras YG, Doğan T, Boncuk S, Eryllmaz HA, et al. Demographic characteristics and neurological comorbidity of patients with COVID-19. Rev Assoc Med Bras (1992). 2020;66(Suppl 2):82-5. https://doi.org/10.1590/1806-9282.66.S2.82

2. Chung MS, Choi WR, Jeong HY, Lee JH, Kim JH. MR Imaging-based evaluations of olfactory bulb atrophy in patients with olfactory dysfunction. AJNR Am J Neuroradiol. 2018;39(3):532-7. https://doi.org/10.3174/ajnr.A5491

3. Buschhüter $D$, Smitka M, Puschmann S, Gerber JC, Witt M, Abolmaali ND, et al. Correlation between olfactory bulb volume and olfactory function. Neuroimage. 2008;42(2):498-502. https://doi.org/10.1016/j.neuroimage.2008.05.004

4. Desforges M, Le Coupanec A, Dubeau P, Bourgouin A, Lajoie $L$, Dubé $M$, et al. Human coronaviruses and other respiratory viruses: underestimated opportunistic pathogens of the central nervous system? Viruses. 2019;12(1):14. https://doi. org/10.3390/v12010014

5. Hopkins C, Surda P, Kumar N. Presentation of new onset anosmia during the COVID-19 pandemic. Rhinology. 2020;58(3):295-8. https://doi.org/10.4193/Rhin20.116

6. Lechien JR, Chiesa-Estomba CM, De Siati DR, Horoi M, Le Bon $S D$, Rodriguez $A$, et al. Olfactory and gustatory dysfunctions as a clinical presentation of mild-to-moderate forms of the coronavirus disease (COVID-19): a multicenter European study. Eur Arch Otorhinolaryngol. 2020;277(8):2251-61. https://doi. org/10.1007/s00405-020-05965-1
7. Nath A. Neurologic complications of coronavirus infections. Neurology. 2020;94(19):809-10. https://doi.org/10.1212/ WNL.0000000000009455

8. Suzuki M, Takashima T, Kadoya M, Takahashi S, Miyayama S, Taira S. MR imaging of olfactory bulbs and tracts. AJNR Am J Neuroradiol. 1989;10(5):955-7. PMID: 2505540

9. Schneider JF, Floemer F. Maturation of the olfactory bulbs: MR imaging findings. AJNR Am J Neuroradiol. 2009;30(6):114952. https://doi.org/10.3174/ajnr.A1501

10. Galougahi MK, Ghorbani J, Bakhshayeshkaram M, Naeini AS, Haseli S. Olfactory bulb magnetic resonance imaging in SARS-CoV-2-induced anosmia: the first report. Acad Radiol. 2020;27(6):892-3. https://doi.org/10.1016/j.acra.2020.04.002

11. Laurendon T, Radulesco T, Mugnier J, Gérault M, Chagnaud C, El Ahmadi AA, et al. Bilateral transient olfactory bulb edema during COVID-19-related anosmia. Neurology. 2020;95(5):224-5. https://doi.org/10.1212NNL.0000000000009850

12. Rombaux P, Mouraux A, Bertrand B, Nicolas G, Duprez T, Hummel T. Olfactory function and olfactory bulb volume in patients with postinfectious olfactory loss. Laryngoscope. 2006;116(3):436-9. https://doi.org/10.1097/01.MLG.0000195291.36641.1E

13. Eliezer $M$, Hautefort $C$, Hamel $A L$, Verillaud $B$, Herman $P$, Houdart $E$, et al. Sudden and complete olfactory loss of function as a possible symptom of COVID-19. JAMA Otolaryngol Head Neck Surg. 2020;146(7):674-5. https://doi.org/10.1001/ jamaoto.2020.0832 
14. Wilson RS, Arnold SE, Schneider JA, Boyle PA, Buchman AS, Bennett DA. Olfactory impairment in presymptomatic Alzheimer's disease. Ann N Y Acad Sci. 2009;1170:730-5. https://doi.org/10.1111/j.1749-6632.2009.04013.x

15. Lund VJ, Kennedy DW. Staging for rhinosinusitis. Otolaryngol Head Neck Surg. 1997;117(3 Pt 2):S35-40. https://doi.org/10.1016/ s0194-5998(97)70005-6

16. Alarabawy RA, Eltomey MA, Shehat EM. Volumetric study of the olfactory bulb in patients with chronic rhinonasal sinusitis using MRI, Egypt. J Radiol Nucl Med. 2016;47(2):487-91. https://doi. org/10.1016/j.ejrnm.2016.02.011

17. Duprez TP, Rombaux P. Imaging the olfactory tract (cranial nerve \#1). Eur J Radiol. 2010;74(2):288-98. https://doi. org/10.1016/j.ejrad.2009.05.065

18. Özkiriş M, Gencer ZK, Saydam L. The Effect of unilateral concha bullosa on olfactory bulb volume: an assessment by magnetic resonance imaging. J Craniofac Surg. 2018;29(2):400-2. https://doi.org/10.1097/SCS.0000000000004086

19. Chung MS, Choi WR, Jeong HY, Lee JH, Kim JH. MR imagingbased evaluations of olfactory bulb atrophy in patients with olfactory dysfunction. AJNR Am J Neuroradiol. 2018;39(3):532-7. https://doi.org/10.3174/ajnr.A5491

20. Tsutsumi S, Ono H, Yasumoto Y. Visualization of the olfactory nerve using constructive interference in steady state magnetic resonance imaging. Surg Radiol Anat. 2017;39(3):315-21. https://doi.org/10.1007/s00276-016-1731-9
21. Yao L, Yi X, Pinto JM, Yuan X, Guo Y, Liu Y, et al. Olfactory cortex and Olfactory bulb volume alterations in patients with post-infectious Olfactory loss. Brain Imaging Behav. 2018;12(5):1355-62. https://doi.org/10.1007/s11682017-9807-7

22. Politi LS, Salsano E, Grimaldi M. Magnetic resonance imaging alteration of the brain in a patient with Coronavirus Disease 2019 (COVID-19) and anosmia. JAMA Neurol. 2020;77(8):1028-9. https://doi.org/10.1001/ jamaneurol.2020.2125

23. van Riel $D$, Verdijk $R$, Kuiken $T$. The olfactory nerve: a shortcut for influenza and other viral diseases into the central nervous system. J Pathol. 2015;235(2):277-87. https://doi.org/10.1002/ path.4461

24. Netland J, Meyerholz DK, Moore S, Cassell M, Perlman $S$. Severe acute respiratory syndrome coronavirus infection causes neuronal death in the absence of encephalitis in mice transgenic for human ACE2. J Virol. 2008;82(15):7264-75. https://doi.org/10.1128/ JVI.00737-08

25. Fodoulian L, Tuberosa J, Rossier D, Boillat M, Kan C, Pauli $V$, et al. SARS-CoV-2 receptors and entry genes are expressed in the human olfactory neuroepithelium and brain. iScience. 2020;23(12):101839. https://doi.org/10.1016/j. isci.2020.101839 